\title{
22q11.2 recurrent copy number variation-related syndrome: a retrospective analysis of our own microarray cohort and a systematic clinical overview of ClinGen curation
}

\author{
Jiangyang Xue ${ }^{1 \#}$, Ru Shen ${ }^{2 \#}$, Min Xie ${ }^{1}$, Yingwen Liu ${ }^{1}$, Yuxin Zhang ${ }^{1}$, Linglu Gong ${ }^{3}$, Haibo Li $^{1 \wedge}$ \\ ${ }^{1}$ The Central Laboratory of Birth Defects Prevention and Control, Ningbo Women and Children's Hospital, Ningbo, China; ${ }^{2}$ Division of \\ Laboratory, Kunming Maternity and Child Care Hospital, Kunming, China; ${ }^{3}$ Ultrasonography Department, Ningbo Women and Children's \\ Hospital, Ningbo, China \\ Contributions: (I) Conception and design: H Li, L Gong; (II) Administrative support: H Li; (III) Provision of study materials or patients: L Gong; (IV) \\ Collection and assembly of data: M Xie, Y Liu, Y Zhang; (V) Data analysis and interpretation: J Xue, R Shen; (VI) Manuscript writing: All authors; (VII) \\ Final approval of manuscript: All authors. \\ \#These authors contributed equally to this work. \\ Correspondence to: Haibo Li. The Central Laboratory of Birth Defects Prevention and Control, Ningbo Women and Children's Hospital, 339 Liuting \\ Street, Ningbo, 315000, China. Email: lihaibo-775@163.com; Linglu Gong. Ultrasonography Department, Ningbo Women and Children’s Hospital, \\ 339 Liuting Street, Ningbo 315000, China. Email: 297547211@qq.com.
}

Background: Chromosomal 22q11.2 dosage changes in the recurrent region can lead to a series of clinically variable pediatric syndromes. This study conducted a retrospective analysis of microarray tested cases with 22q11.2 recurrent copy number variations (CNVs) at our laboratory from September 2018 to August 2021, and provides a systematical clinical overview of ClinGen curation.

Methods: The data of 34 microarray tested cases with 22q11.2 recurrent CNVs at our laboratory from September 2018 to August 2021 were retrospectively analyzed, and the variant types, abnormal chromosome regions, clinical phenotypes, and follow-up information were evaluated and summarized. A ClinGen Dosage Sensitivity Map was retrieved for " $22 \mathrm{q} 11.2$ ". The information of each $22 \mathrm{q} 11.2$ recurrent region was collected and systematically classified.

Results: We reported 34 cases (including 18 22q11.2 microdeletion cases and 16 microduplication cases) from 8,465 microarrays. Of the 22q11.2 recurrent CNV-carried samples, 74\% (25/34) comprised prenatal amniotic fluid or villus, and up to $50 \%$ (17/34) of the cases contained the proximal A-D interval. Across these 22q11.2 microdeletion samples, the congenital cardiovascular defect, which mainly included the tetralogy of fallot, ventricular septal defect, and patent foramen ovale, was identified as the most common feature (13/18, $72 \%$ ). However, 22q11.2 microduplication cases exhibited a broad range of highly variable phenotypes, spanning from severe abnormality to mild characteristics and even the completely normal phenotype. This study also systematically reviewed the ClinGen dosage sensitivity curation on 22q11.2 recurrent regions, and found that A-D/A-B haploinsufficiency score reached "3", responsible for DiGeorge syndrome (DGS)/ velocardiofacial syndrome (VCFS). Also, A-D/A-B triplosensitivity score "3" could further account for multiple variable phenotypes.

Conclusions: Taken together, this study provides clinical overview of the ClinGen curation and data support for the American College of Medical Genetics and Genomics (ACMG) evaluation in the pathogenicity of each interval involved in 22q11.2 recurrent deletion and duplication. Certainly, more evidences on the genotype-phenotype contributions of different 22q11.2 recurrent CNVs need to be gathered.

Keywords: 22q11.2 recurrent copy number variations (22q11.2 recurrent CNVs); retrospective analysis; overview; ClinGen curation

\footnotetext{
$\wedge$ ORCID: 0000-0002-9309-6632.
} 
Submitted Oct 26, 2021. Accepted for publication Dec 15, 2021.

doi: $10.21037 / \mathrm{tp}-21-560$

View this article at: https://dx.doi.org/10.21037/tp-21-560

\section{Introduction}

A cluster of low-copy repeats (LCRs) from A-H in chromosome 22q11.2 (chr22:17,900,001-25,900,000), also known as LCR22A-H, mediate nonallelic homologous recombination and cause 22 q 11.2 chromosomal rearrangements. Various intervals occur during this process that are respectively described as "proximal," "central," and "distal" on the basis of the corresponding length and region of the recurrent copy number variations (CNVs) $(1,2)$. Both the recurrent regions [(i.e., $3 \mathrm{Mb}$ (A-D: chr22:18,912,231$21,465,672)$ and $1.5 \mathrm{Mb}(\mathrm{A}-\mathrm{B}: \operatorname{chr} 22: 18,912,231-$ $20,287,208)]$ included in the proximal CNVs are the most common types for $22 \mathrm{q} 11.2$ microdeletions (the small deleted $\mathrm{CNVs}$ ) or microduplications (the small duplicated $\mathrm{CNVs}$ ). As previously reported, the proximal A-D or AB deletion may lead to severe DiGeorge syndrome (DGS)/ velocardiofacial syndrome (VCFS) (3), while identical duplication results in highly variable and nonspecific phenotypes (4).

The $22 \mathrm{q} 11.2$ central and distal CNV syndromes also express phenotypic variability, spanning from severe abnormality to mild characteristics and even a completely normal phenotype $(5,6)$. This creates great challenges for genetic counseling and the prediction of clinical consequences. Just recently, the prenatal ultrasound phenotypes of CNVs in different regions of 22q11.2, their parental original, as well as pregnancy outcome, were analyzed and reported by Peixuan group. Interestingly, they found that prenatal phenotypes of the $22 \mathrm{q} 11.2$ region $\mathrm{CNV}$ are diverse, which may be related to gene function; nuchal translucency (NT) thickening may be used as an early ultrasound finding of proximal 22q11.2 CNV (7). In this study, we retrospectively analyzed 34 microarray cases with $22 \mathrm{q} 11.2$ recurrent $\mathrm{CNVs}$ at our own laboratory from September 2018 to August 2021, and systematically reviewed each recurrent $22 \mathrm{q} 11.2$ region for which the ClinGen expert group had completed curation (derived from the ClinGen Dosage Sensitivity Map that ClinGen consortium curates genes and regions of the genome to assess whether there is evidence to support that these genes/ regions are dosage sensitive and should be targeted on a cytogenomic array). We present the following article in accordance with the STROBE reporting checklist (available at https://dx.doi.org/10.21037/tp-21-560).

\section{Methods}

\section{Collection of clinical information}

We undertook a retrospective analysis of 34 cases of 22 q 11.2 recurrent CNVs (including 18 22q11.2 microdeletion cases and 16 microduplication cases) from 8465 microarrays performed at our own laboratory from September 2018 to August 2021. Prenatal amniotic fluid and villus, abortion tissue and villus, as well as peripheral blood derived from children/adults were used in our analysis. Data on gestation/age, the chromosome microarray analysis (CMA) results and corresponding variant evaluation types, clinical phenotypes (prenatal samples conforming to B ultrasound data), and tests were collected and are summarized in Table 1 . After post-test counseling, a fraction of parents received a CMA analysis to validate their fetuses' hereditary mode. Additionally, 5 months after CMA testing, the pregnant women and probands were followed up with by telephone. All the available information is displayed in Table 1. "N/ A" represents a loss of communication, an unwillingness to inform, or an unknown hereditary mode. Written informed consent was provided by each pregnant woman, proband, or their parents. The study was approved by ethics board of Ningbo Women and Children's Hospital (No. EC2020014). This study conformed to the provisions of the Declaration of Helsinki (as revised in 2013).

\section{$C M A$}

After samples were collected, DNA was extracted using a TIANamp Genomic DNA Kit (Cat.\#DP304-02; Lot\#U8420). Then, 250ng DNA was amplified, labeled, and hybridized to the GCS 3000Dx v.2 platform (Affymetrix, Santa Clara, CA, USA). The SNP array test was processed with a commercial $750 \mathrm{~K}$ microarray chip (CytoScan $750 \mathrm{~K}$ Array; Affymetrix). The chip was washed with buffer, scanned with a laser scanner after hybridization with fragmented DNA. The data were analyzed using Chromosome Analysis Suite v3.2 (Affymetrix). 
Table 1 The clinical information of 34 cases carried with $22 q 11.2$ recurrent CNVs

\begin{tabular}{|c|c|c|c|c|c|c|c|c|c|}
\hline $\begin{array}{l}22911.2 \\
\text { CNV type }\end{array}$ & $\begin{array}{l}\text { Case } \\
\text { number }\end{array}$ & $\begin{array}{l}\text { Sample } \\
\text { type }\end{array}$ & $\begin{array}{l}\text { Gestation/ CMA analysis result } \\
\text { age }\end{array}$ & $\begin{array}{l}\text { Variant } \\
\text { evaluation type }\end{array}$ & 22911.21 recurrent region & Clinical phenotypes and tests (contain ultrasound data) & Follow-up information & $\begin{array}{l}\text { Parental validation } \\
\text { (yes/not) }\end{array}$ & Hereditary mode \\
\hline \multirow[t]{17}{*}{ Microdeletion } & 1 & $\begin{array}{l}\text { Amniotic } \\
\text { fluid }\end{array}$ & $20 W+6 D$ arr[hg19] 22q11.21(20,716,876-21,800,471) ×1 & $\begin{array}{l}\text { Likely } \\
\text { pathogenic }\end{array}$ & Contain central (B-D) region & $\begin{array}{l}\text { NIPT: a microdeletion of chromosome 22; B ultrasound: strong light } \\
\text { spots in the left ventricle, patent foramen ovale, oligohydramnios }\end{array}$ & $\begin{array}{l}\text { Premature infant, } \\
\text { anemiaofprematurity, } \\
\text { neonatal respiratory } \\
\text { distress syndrome, } \\
\text { neonatal pneumonia, } \\
\text { low birth weight infant }\end{array}$ & No & N/A \\
\hline & 2 & & arr[hg19] 22q11.22q11.23(22,997,928-23,654,007) ×1 & $\begin{array}{l}\text { Likely } \\
\text { pathogenic }\end{array}$ & Contain distal type II (E-F) region & Advanced maternal age & $\mathrm{N} / \mathrm{A}$ & Yes & Inherited from mother \\
\hline & 3 & & $25 W+2 D \operatorname{arr}[h \operatorname{hig}] 22 q 11.21(20,716,876-21,800,471) \times 1$ & Pathogenic & Contain central (B-D) region & $\begin{array}{l}\text { NIPT: a local microdeletion of chromosome 22; B ultrasound: patent } \\
\text { foramen ovale }\end{array}$ & $\mathrm{N} / \mathrm{A}$ & Yes & Inherited from mother \\
\hline & 4 & & $26 W+3 D$ arr[hg19] 22q11.21(18,916,842-21,800,471) ×1 & Pathogenic & Contain proximal (A-D) region & $\begin{array}{l}\text { B ultrasound: congenital heart disease (perimembrane ventricular septal } \\
\text { defect) }\end{array}$ & N/A & Yes & De novo \\
\hline & 5 & & $19 W+2 D$ arr[hg19] 22q11.21(18,916,842-21,800,471) $\times 1$ & Pathogenic & Contain proximal (A-D) region & $\begin{array}{l}\text { The histories of abnormal pregnancy: gave birth to a child with tetralogy } \\
\text { of fallot; B ultrasound: complicated congenital heart disease (tetralogy of } \\
\text { fallot + pulmonary atresia), the absent thymus }\end{array}$ & N/A & Yes & Inherited from mother \\
\hline & 6 & & $19 W+6 D \operatorname{arr}[h \operatorname{hg} 19] 22 q 11.21(20,716,876-21,800,471) \times 1$ & vus & Contain central (B-D) region & NIPT: a microdeletion of chromosome 22 & $\mathrm{~N} / \mathrm{A}$ & Yes & Inherited from mother \\
\hline & 7 & & $\operatorname{arr}[$ hg19] 22q11.21(18,919,477-21,800,471) ×1 & Pathogenic & Contain proximal $(A-D)$ region & $\begin{array}{l}\text { Advanced maternal age; NIPT: a microdeletion of chromosome 22; B } \\
\text { ultrasound: complicated congenital heart disease (tetralogy of fallot) }\end{array}$ & $\mathrm{N} / \mathrm{A}$ & No & N/A \\
\hline & 8 & & $\operatorname{arr}[$ hg19] 22q11.21(18,919,477-21,800,471) ×1 & Pathogenic & Contain proximal (A-D) region & $\begin{array}{l}\text { NIPT: } 5 \text { Mb deletion in 22q11.1911.21; B ultrasound: fetal vagus right } \\
\text { subclavian artery }\end{array}$ & $\mathrm{N} / \mathrm{A}$ & Yes & De novo \\
\hline & 10 & & $\operatorname{arr}[$ hg19] 22q11.21(18,919,478-21,058,888) ×1 & Pathogenic & Overlap with proximal (A-D) region & $\begin{array}{l}\text { B ultrasound: right aortic arch, strong light spot in right ventricle, slightly } \\
\text { enlarged right atrium }\end{array}$ & Embryo arrest at $28 \mathrm{~W}$ & Yes & De novo \\
\hline & 11 & & $20 \mathrm{~W}+4 \mathrm{D}$ arr[hg19] 22q11.21q11.22(21,917,140-22,962,962) $\times 1$ & Pathogenic & Overlap with distal type I (D-E/F) & NIPT suggested microdeletion on chromosome 22 & $\mathrm{~N} / \mathrm{A}$ & No & N/A \\
\hline & 12 & $\begin{array}{l}\text { Peripheral } \\
\text { blood }\end{array}$ & $\operatorname{arr}[$ hg19] 22q11.21(18,919,477-21,800,471) ×1 & Pathogenic & Contain proximal (A-D) region & $\begin{array}{l}\text { Multiple malformations of abnormal sexual development (micropenis, } \\
\text { microrchidia), intellectual/physical retardation and congenital heart } \\
\text { disease }\end{array}$ & $\mathrm{N} / \mathrm{A}$ & No & N/A \\
\hline & 13 & $\begin{array}{l}\text { Abortion } \\
\text { tissue }\end{array}$ & arr[hg19] 22q11.21(18,648,855-21,915,207) ×1 & Pathogenic & Contain proximal (A-D) region & $\begin{array}{l}\text { B ultrasound: the possible pulmonary dysplasia with atresia and severe } \\
\text { stenosis, severe tetralogy of fallot }\end{array}$ & Odinopoeia & No & N/A \\
\hline & 14 & & $22 W+3 D \operatorname{arr}[h \operatorname{h19}] 22 q 11.21(19,024,793-21,800,471) \times 1$ & Pathogenic & Contain proximal (A-D) region & $\begin{array}{l}\text { B ultrasound: persistent truncus arteriosus, ventricular septal defect } \\
\text { (malalignment type) }\end{array}$ & Odinopoeia & Yes & De novo \\
\hline & 15 & & $20 W+4 D$ arr[hg19] 22q11.21(18,648,855-21,800,471) ×1 & Pathogenic & Contain proximal (A-D) region & $\begin{array}{l}\text { B ultrasound: ventricular septal defect, aortic riding, pulmonary stenosis, } \\
\text { suggesting tetralogy of fallot; PLSVC }\end{array}$ & Odinopoeia & Yes & De novo \\
\hline & 16 & & $\begin{array}{r}17 \mathrm{~W}+2 \mathrm{2D} \begin{array}{r}\operatorname{arr}[\mathrm{hg} 19] 3 \mathrm{3q} 26.1(161,044,139-161,888,507) \times 3 \\
\quad \operatorname{arr}[\mathrm{hg} 19] \\
22 \mathrm{q} 11.21(21,058,887-21,800,471) \times 1\end{array}\end{array}$ & Pathogenic & Overlap with proximal (A-D) region & $\begin{array}{l}\text { B ultrasound: ventricular septal defect, aortic riding, pulmonary stenosis, } \\
\text { suggesting tetralogy of fallot; PLSVC }\end{array}$ & Odinopoeia & No & N/A \\
\hline & 17 & & $\operatorname{arr}[$ hg19] 22q11.21(18,648,856-21,800,471) ×1 & Pathogenic & Overlap with proximal (A-D) region & Congenital heart disease, left kidney dysplasia & $\mathrm{N} / \mathrm{A}$ & No & N/A \\
\hline & 18 & $\begin{array}{l}\text { Abortion } \\
\text { villus }\end{array}$ & $\operatorname{arr}[$ hg19] 22q11.21(18,919,478-21,800,471) $\times 1$ & Pathogenic & Overlap with proximal $(A-D)$ region & NT thickening; lymphatic hydrocystic tumor & N/A & No & N/A \\
\hline
\end{tabular}

Table 1 (continued) 
Table 1 (continued)

\begin{tabular}{|c|c|c|c|c|c|c|c|c|c|c|}
\hline $\begin{array}{l}22 q 11.2 \\
\text { CNV type }\end{array}$ & $\begin{array}{l}\text { Case } \\
\text { number }\end{array}$ & 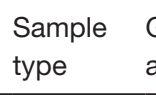 & $\begin{array}{l}\text { Gestation/ } \\
\text { age }\end{array}$ & CMA analysis result & $\begin{array}{l}\text { Variant } \\
\text { evaluation type }\end{array}$ & $22 q 11.21$ recurrent region & Clinical phenotypes and tests (contain ultrasound data) & Follow-up information & $\begin{array}{l}\text { Parental validation, } \\
\text { (yes/not) }\end{array}$ & Hereditary mode \\
\hline \multirow[t]{16}{*}{ Microduplication } & 19 & $\begin{array}{l}\text { Amniotic } 1 \\
\text { fluid }\end{array}$ & $19 W+4 D a$ & arr[hg19] 22q11.21(18,648,855-21,800,471) ×3 & Pathogenic & Contain proximal (A-D) region & The abnormal prenatal BoBs result: 22q11.2 microduplication & N/A & No & N/A \\
\hline & 20 & & $22 W+1 D a$ & $\begin{array}{l}\text { arr[hg19] 7q11.23(72,624,166-74,197,150) } \times 1 ; \\
\text { arr[hg19] 22q11.21(18,648,855-21,800,471) ×3 }\end{array}$ & Pathogenic & Contain proximal (A-D) region & $\begin{array}{l}\text { Advanced maternal age; B ultrasound: ventricular septal defect, the } \\
\text { increased S/D ratio of umbilical artery blood flow, undetected right } \\
\text { kidney }\end{array}$ & $\begin{array}{l}\text { Postpartum neonatal } \\
\text { jaundice }\end{array}$ & No & N/A \\
\hline & 21 & & $21 W+5 D a$ & arr[hg19] 22q11.21(18,970,561-21,800,471) ×3 & Pathogenic & Contain proximal (A-D) region & Fetal serological screening: the high risk of 21 trisomy & N/A & Yes & De novo \\
\hline & 22 & & $21 W+4 D a$ & arr[hg19] 22q11.21(18,648,855-21,459,713) ×3 & Pathogenic & Contain proximal $(A-D)$ region & $\begin{array}{l}\text { NIPT: a local microduplication of chromosome 22; B ultrasound: } \\
\text { oligohydramnios }\end{array}$ & N/A & No & N/A \\
\hline & 23 & & $26 \mathrm{~W}+6 \mathrm{D} a$ & $\operatorname{arr}[$ hg19] 22q11.21(18,648,855-21,800,471) ×3 & Pathogenic & Contain proximal (A-D) region & B ultrasound: fetal cerebral ventriculomegaly: $11 \mathrm{~mm}$ & N/A & Yes & Inherited from father \\
\hline & 24 & & $19 W+4 D a$ & arr[hg19] 22q11.21(20,716,902-21,461,017) ×3 & vus & Contain central (B-D) region & $\begin{array}{l}\text { Fetal serological screening: the high risk of } 21 \text { trisomy; } \\
\text { NT thickening: } 2.8 \mathrm{~mm}\end{array}$ & N/A & No & N/A \\
\hline & 25 & & $19 W+4 D a$ & arr[hg19] 22q11.23(23,692,307-24,987,835) ×3 & vus & Contain distal type III (F-G) region & Fetal serological screening: the high risk of 21 trisomy & N/A & No & N/A \\
\hline & 26 & & $20 W+1 D a$ & $\operatorname{arr}[$ hg19] 22q11.21(18,919,477-21,800,471) ×3 & Pathogenic & Contain proximal (A-D) region & $\begin{array}{l}\text { Advanced maternal age; the pregnant woman with macular degeneration } \\
\text { and neurodeatrophia; B ultrasound: oligohydramnios }\end{array}$ & $\begin{array}{l}\text { Postpartum B } \\
\text { ultrasound: patent } \\
\text { foramen ovale; } \\
\text { neonatal dyspnea } \\
\text { syndrome; neonatal } \\
\text { pneumonia }\end{array}$ & No & N/A \\
\hline & 27 & & $24 \mathrm{~W}$ & $\operatorname{arr}[$ hg19] 22q11.21(18,916,960-21,800,471) ×3 & Pathogenic & Contain proximal (A-D) region & $\begin{array}{l}\text { The histories of abnormal pregnancy: gave birth to a retarded child with } \\
\text { her ex-husband; B ultrasound: a fissure is seen in the gall bladder, strong } \\
\text { light spots in the left ventricle }\end{array}$ & N/A & No & N/A \\
\hline & 28 & & $\begin{array}{rl}19 W+2 D & a \\
a & \end{array}$ & 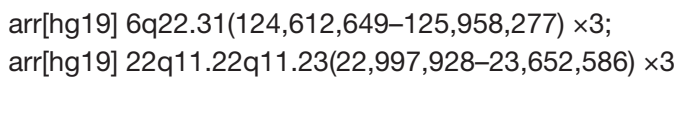 & Likely Benign & Contain distal type II $(E-F)$ region & $\begin{array}{l}\text { The histories of abnormal pregnancy: labor induction for long bone } \\
\text { dysplasia; abnormal 6q22.31, 22q11.22q11.23 in the first fetus and the } \\
\text { mother }\end{array}$ & N/A & Yes & Inherited from mother \\
\hline & 29 & & $20 \mathrm{~W}$ & 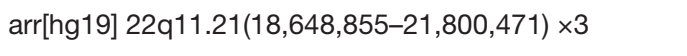 & Pathogenic & Contain proximal $(A-D)$ region & Fetal serological screening: the high risk of 21 trisomy & N/A & No & N/A \\
\hline & 30 & & $21 W+2 D a$ & arr[hg19] 22q11.21(18,916,842-21,800,471) ×3 & Pathogenic & Contain proximal $(A-D)$ region & $\begin{array}{l}\text { Fetal serological screening: the high risk of } 21 \text { trisomy; B ultrasound: the } \\
\text { small and obscured transparent diaphragmatic cavity }\end{array}$ & N/A & No & N/A \\
\hline & 31 & & $20 \mathrm{~W}+4 \mathrm{D} a$ & arr[hg19] 22q11.21(18,919,478-21,800,471) ×3 & Pathogenic & Overlap with proximal (A-D) region & Fetal serological screening at high risk, DS:1/236 & N/A & No & N/A \\
\hline & 32 & $\begin{array}{l}\text { Prenatal } \\
\text { fluff }\end{array}$ & $12 W+3 D a$ & arr[hg19] 22q11.21(18,648,856-21,800,471) ×3 & Pathogenic & Overlap with proximal $(A-D)$ region & NT thickening: $3.7 \mathrm{~mm}$; embryo stop once & N/A & No & N/A \\
\hline & 33 & $\begin{array}{l}\text { Peripheral } 3 \\
\text { blood }\end{array}$ & & $\operatorname{arr}[$ hg19] 22q11.23(23,652,586-25,059,827) ×3 & Vus & Contain distal type III (F-G) region & $\mathrm{N} / \mathrm{A}$ & N/A & No & N/A \\
\hline & 34 & $\begin{array}{l}\text { Abortion } 2 \\
\text { tissue }\end{array}$ & $25 W+3 D a$ & $\begin{array}{l}\text { arr[hg19] 6q22.31(124,612,649-125,928,351) ×3; } \\
\text { arr[hy19] 22q11.22q11.23(22,997,928-23,650,873) ×3 }\end{array}$ & vus & Contain distal type II $(E-F)$ region & $\begin{array}{l}\text { The previous microarry result of aborted fetal tissue: arr[hg19] } \\
6 q 22.31(124,612,649-125,928,351) \times 3 \text {; arrhhg19] } 22911.2 \\
\text { 2q11.23(22,997,928-23,650,873) } 33 \text {; B ultrasound: short limbs long-bone, } \\
\text { slightly weakened echo of spine and other whole body bone }\end{array}$ & N/A & Yes & Inherited from mother \\
\hline
\end{tabular}

CNVs, copy number variations; W, weeks; D, days; $\mathrm{Y}$, years; NIPT, noninvasive prenatal testing; NT, nuchal translucency; VUS, variant of uncertain significance; PLSVC, perpetuate left superior vena cava; S/D, systolic/diastolic. 


\section{Overview of ClinGen curation}

A ClinGen Dosage Sensitivity Map was retrieved for " $22 \mathrm{q} 11.2$ ". The information of each $22 \mathrm{q} 11.2$ recurrent region was collected. The score of each interval's haploinsufficiency/triplosensitivity in current ClinGen curation, and respective clinical features, hereditary mode, and penetrance were all systematically classified and are summarized in Table 2.

\section{Statistical analysis}

The data were analyzed using Microsoft Excel 2010, and not involved in complex statistical analysis.

\section{Results}

\section{Case presentation of $22 q 11.2$ recurrent $C N V s$}

We performed a retrospective analysis of 8,465 microarray cases at our laboratory from September 2018 to August 2021. We identified 34 patients carrying 22 q 11.2 recurrent CNVs (including 18 microdeletion cases and 16 microduplication cases), and examined their genotypephenotype correlations. Their detailed clinical information is summarized in Table 1. Similar to previous reports, the $22 \mathrm{q} 11.2 \mathrm{CNV}$ s containing the proximal A-D interval comprised the most common recurrent region in our CMA data $(17 / 34,50 \%)$. In addition, the aberrant 22q11.2 CNVs of the other 6 cases (including 4 deleted fragments and 2 duplicated fragments) overlapped with the A-D region (6/34, 17.6\%). As expected, all of these 22q11.2 CNVs were assessed as pathogenic variants. The central B-D interval (chr22:20,731,986-21,465,672) was found to be involved in the deletion/duplication regions of 5 cases (5/34, 14.7\%). Additionally, 6 distal 22q11.2 CNVs were found to contain type II (E-F) $(3 / 34,8.8 \%)$ and type III (F-G) $(2 / 34,5.9 \%)$ regions and overlap with the type I (D-E/F) (1/34, 2.9\%) interval. Notably, partially due to their variable phenotypes, incomplete penetrance, low dosage, or incomplete sensitivity curation, the majority of these CNVs were evaluated as variants of uncertain significance according to the American College of Medical Genetics and Genomics (ACMG) criteria.

Our cases were also accompanied by clinical phenotypes (ultrasound data), fractional follow-up information, and hereditary mode data. Across these 22q11.2 microdeletion samples, the congenital cardiovascular defect was identified as the most common feature $(13 / 18,72 \%)$, and mainly included tetralogy of fallot, ventricular septal defect, and patent foramen ovale. In particular, the pathogenic microdeletion variants concerning these congenital heart diseases generally spanned the proximal A-D interval. Most of the samples involved comprised prenatal amniotic fluid, so the clinical characteristics of the fetus were mainly distinguished by a B ultrasound combined with postnatal follow-up data. First, by detection with amniotic fluid, we exemplified cases 1 and 5 . In the prenatal B ultrasound of the case 1 fetus with central B-D deletion, we observed strong light spots in the left ventricle and a patent foramen ovale. The postnatal follow-up information revealed more severe features, including a premature infant, anemia of prematurity, neonatal respiratory distress syndrome, neonatal pneumonia, and a low birth weight. In case 5 , the pregnant woman had ever before given birth to an abnormal child with tetralogy of fallot. Unfortunately, the B ultrasound of the case 5 fetus indicated a more complicated congenital heart disease (tetralogy of fallot and pulmonary atresia) combined with an absent thymus, but the validated parental result suggested that the proximal A-D CNV of case 5 was inherited from the mother, despite the fact that $>90 \%$ A-D deleted cases are de novo (3).

Among the 18 microdeletion cases, case 12 was a 10 -yearold child who was confirmed to carry a pathogenic A-D deletion that was responsible for his multiple malformations of abnormal sexual development (i.e., a micropenis and microrchidia), intellectual/physical retardation, and congenital heart disease. Except for amniotic fluid and peripheral blood detections, the microdeletion CMA data of the abortion tissues and abortion villi are also set out in Table 1. As we observed, cases 13-15 contained the proximal (AD) region and cases 16-17 overlapped with the proximal (AD) region, and all of these cases presented with complicated congenital heart disease, including a severe tetralogy of fallot, ventricular septal defect, persistent truncus arteriosus, or perpetuate left superior vena cava (PLSVC). Parental validation of cases 14 and 15 indicated de novo variants.

Consistent with ClinGen triplosensitivity phenotype comments, the $22 \mathrm{q} 11.2$ recurrent microduplications exhibited a broad range of highly variable phenotypes. Of these affected amniotic fluid cases, $46 \%$ of the pregnant women primarily sought CMA help due to the high risk of 21 trisomy in fetal serological screening. The B ultrasound results revealed multiple variable phenotypes, including a ventricular septal defect, undetected right kidney, fetal cerebral ventriculomegaly, fissure in the gall bladder, and small and obscured transparent diaphragmatic 
Table 2 The systematical clinical overview of ClinGen curation on 22q11.2 recurrent CNVs

\begin{tabular}{|c|c|c|c|c|c|c|c|c|c|}
\hline \multirow{3}{*}{$22 q 11.21$ recurrent region } & \multirow{3}{*}{ Overlap } & \multicolumn{8}{|c|}{ Contained } \\
\hline & & \multicolumn{2}{|c|}{ Proximal } & \multicolumn{2}{|l|}{ Central } & \multicolumn{4}{|c|}{ Distal } \\
\hline & & $A-B$ & $A-D$ & $B-D$ & C-D & Type I (D-E/F) & Type II (E-F) & Type III (D-H) & Type III (F-G) \\
\hline Region location (GRCh37) & chr22:17,392,953-18,591,860 & chr22:18,912,231-20,287,208 & chr22:18,912,231-21,465,672 & chr22:20,731,986-21,465,672 & $\begin{array}{c}\text { chr22:21,092,338- } \\
21,465,672\end{array}$ & chr22:21,917,117-23,649,111 & $\begin{array}{c}\operatorname{chr} 22: 23,119,414- \\
23,649,111\end{array}$ & $\begin{array}{c}\text { chr22:21,917,117- } \\
24,994,433\end{array}$ & $\begin{array}{c}\text { chr22:23,831,202- } \\
24,632,821\end{array}$ \\
\hline Key morbid genes & CECR2 & & $B X 1$ & CRKL & & N/A & N/A & SMA & CCB1 \\
\hline \multicolumn{10}{|c|}{ The score of dosage sensitivity in ClinGen gene curation } \\
\hline Haploinsufficiency & 0 & 3 & 3 & 2 & N/A & 3 & N/A & N/A & N/A \\
\hline Triplosensitivity & 3 & 3 & 3 & 1 & N/A & 3 & N/A & N/A & N/A \\
\hline \multicolumn{10}{|l|}{ Clinical features } \\
\hline Haploinsufficiency & $\begin{array}{l}\text { Rare in both the literature and } \\
\text { databases of genomic variation } \\
\text { (both clinical and control } \\
\text { populations) }\end{array}$ & $\begin{array}{l}\text { DGS/NCFS syndrome, congen } \\
\text { characteristic facial features, D } \\
\text { deficiency, hypocalcemia }\end{array}$ & $\begin{array}{l}\text { heart disease, palatal abnormalities, } \\
\text { D, behavior problems, immune }\end{array}$ & $\begin{array}{l}\text { Phenotypic variability include: } \\
\text { dysmorphic facial features, growth } \\
\text { restriction/short stature, CNS } \\
\text { anomalies/seizures, developmental } \\
\text { delay (including language delay), } \\
\text { intellectual disability, psychiatric/ } \\
\text { behavioral problems, skeletal } \\
\text { anomalies, cardiovascular defects, } \\
\text { genitourinary anomalies, and } \\
\text { immune deficiency/recurrent } \\
\text { infections }\end{array}$ & N/A & $\begin{array}{l}\text { Phenotypic variability include: } \\
\text { preterm birth, pre- and/or } \\
\text { postnatal growth restriction, } \\
\text { DD/ID, behavioral problems, } \\
\text { cardiovascular defects, skeletal } \\
\text { anomalies and mild dysmorphic } \\
\text { facial features }\end{array}$ & N/A & N/A & N/A \\
\hline Triplosensitivity & CES, phenotypic variability & $\begin{array}{l}\text { Highly variable clinical phenoty } \\
\text { expression a broad range of cli } \\
\text { phenotypes or phenotypes tha }\end{array}$ & $\begin{array}{l}\text { ranging from apparently normal to } \\
\text { al features, including nonspecific } \\
\text { verlap clinical findings of DGS/NCFS }\end{array}$ & Phenotypic variability & N/A & $\begin{array}{l}\text { Phenotypic variability include: } \\
\text { developmental delays and facial } \\
\text { dysmorphisms }\end{array}$ & N/A & N/A & N/A \\
\hline \multicolumn{10}{|l|}{ Hereditary mode } \\
\hline Haploinsufficiency & N/A & $>90 \%$ are de novo & & $60 \%$ are de novo & N/A & The majority are de novo & N/A & N/A & N/A \\
\hline Triplosensitivity & N/A & Frequently inherited & & $\mathrm{N} / \mathrm{A}$ & N/A & $\mathrm{N} / \mathrm{A}$ & N/A & N/A & N/A \\
\hline \multicolumn{10}{|l|}{ Penetrance } \\
\hline Haploinsufficiency & $\mathrm{N} / \mathrm{A}$ & Enriched in the clinical populat & & Incomplete & $\mathrm{N} / \mathrm{A}$ & Enriched in the clinical population & N/A & N/A & N/A \\
\hline Triplosensitivity & N/A & Incomplete, enriched in the clir & a population & Incomplete & N/A & $\begin{array}{l}\text { Incomplete, enriched in the clinical } \\
\text { population }\end{array}$ & N/A & N/A & N/A \\
\hline
\end{tabular}

CNVs, copy number variations; CES, cat eye syndrome; DGS/NCFS, DiGeorge syndrome/velocardiofacial syndrome; DD/ID, developmental delay/intellectual disability; CNS, central nervous system. 
cavity. Another abortion tissue with a duplicated variant of uncertain significance (VUS) inherited from the mother contained a distal type II (E-F) region, and presented with shorter long bones of the limbs, a slightly weakened echo of the spine and other body bones.

\section{Clinical overview of the ClinGen curation}

The proximal $\mathrm{A}-\mathrm{D} / \mathrm{A}-\mathrm{B}$ recurrent $\mathrm{CNV}$ s are responsible for the most common microdeletions or microduplications in chromosome 22q11.2. In particular, A-D/A-B regional deletions can lead to severe DGS/VCFS syndrome for which clinical phenotypes typically include congenital heart disease (particularly conotruncal malformations), palatal abnormalities (particularly velopharyngeal incompetence, cleft palate, and bifid uvula), characteristic facial features, developmental delay and intellectual disability (DD/ID), behavior problems, immune deficiency, and hypocalcemia $(3,8)$. The score of haploinsufficiency sensitivity in the ClinGen gene curation reached " 3 ". Among the 30 involved protein coding genes, TBX1 was identified as the most crucial morbid gene for DGS/VCFS syndrome. Yagi et al. found that a heterozygous 1 base pair deletion (1223delC) in the TBX1 gene caused a frameshift leading to a stop codon, and thus induced conotruncal anomaly face syndrome/ VCFS (9). Paylor et al. reported that a heterozygous 23 base pair deletion (1320-1342del23) in the TBX1 gene could result in a frameshift and the extension of the protein from 504 to 616 amino acids, for which a mutation was found in a mother and her 2 sons who presented with VCFS (10). Nevertheless, patients with proximal duplication in 22q11.2 share a more variable clinical phenotype, ranging from apparently normal to nonspecific phenotypes (e.g., intellectual disability, learning disability, developmental delays, autism, psychiatric disorder growth delays, and hypotonia) $(2,4)$. According to the literature, $>90 \% 22 \mathrm{q} 11.2$ proximal (DGS/VCFS) deletions are de novo (3), while AB/A-D duplications are frequently inherited (4). Notably, cat eye syndrome (CES) results from the tetrasomy/ triplication of the CES critical region (CESCR) that overlaps with the chromosome 22q11.2's proximal region. The CESCR includes the CECR 1 and CECR 2 genes, which are responsible for heart/facial and neurologic/eye features, respectively, but does not involve the DGS/VCFS syndrome region of $22 \mathrm{q} 11.2(11,12)$.

$22 \mathrm{q} 11.2$ central recurrent $\mathrm{CNV}$ s contain B-D and C$\mathrm{D}$ regions. The current haploinsufficiency score of $\mathrm{B}-$ $D$ reaches " 2 " for the emerging evidence of phenotypic variability, including dysmorphic facial features, growth restriction/short stature, central nervous system (CNS) anomalies/seizures, developmental delay (including language delay), intellectual disability, psychiatric/ behavioral problems, skeletal anomalies, cardiovascular defects, genitourinary anomalies, and immune deficiency/ recurrent infections. Among the collected cases, $60 \%$ of the deletions were confirmed to be de novo events by parental testing $(1,5,13)$. Similarly, the current triplosensitivity score for the B-D region is only "1" due to the limited number of patients reported in the literature, phenotypic variability, incomplete penetrance, and the lack of case-controlled data.

The distal CNVs of 22q11.2 mainly include 3 types, and currently, only the haploinsufficiency and triplosensitivity of type I (D-E/F) have been curated. ClinGen experts summarized the variable clinical phenotypes of D-E/ F deletion: preterm birth, pre- and/or postnatal growth restriction, DD/ID, behavioral problems, cardiovascular defects, skeletal anomalies, and mild dysmorphic facial features. The deletions are de novo events in the majority of cases, but occasionally a few carrier parents are reported to have a mild or normal phenotype $(14,15)$. Conversely, the variable clinical phenotypes of D-E/F microduplication mainly include developmental delays and facial dysmorphisms that exhibit incomplete penetrance in the clinical population $(16,17)$. The above contents are included in the ClinGen dosage sensitivity curation page, and have been further summarized in Table 2. The dosage sensitivity curation of type II (E-F), type III (D-H or FG) CNVs have not been completed, but sporadic cases have been reported.

\section{Discussion}

Multiple studies have conducted systematic reviews of 22q11.2 microdeletion/microduplication syndrome, performed statistical analyses of their own CMA data and elucidated possible genotype-phenotype contributions (18-20). For example, Pinchefsky et al. reported on the clinical phenotype and cytogenetic studies of a 3-year-old girl with a de novo distal 22q11.2 duplication, reviewed the literature associated with distal 22q11.2 duplication, and compared the clinical features of 28 previously published cases (6). Burnside's review article on Gytogenetic and Genome Research systematically classified each the CNV of $22 \mathrm{q} 11.2$ region and provided a detailed overview of patients' clinical features (as available in the literature). Additionally, their own cohort of postnatal and prenatal microarray cases 
with the $22 \mathrm{q} 11.2 \mathrm{CNV}$ abnormality were also included in their review (1). Emerging reports of patients with $22 \mathrm{q} 11.2$ variants in the literature provide favorable evidence for the ClinGen dosage sensitivity curation experts, who are curating genes and regions of the genome to assess whether there is evidence that these genes/regions are dosage sensitive. The score of haploinsufficiency or triplosensitivity in ClinGen gene curation could further support the pathogenicity evaluation of chromosome CNVs.

According to the ClinGen dosage sensitivity curation and clinical overview of $22 \mathrm{q} 11.2$ recurrent $\mathrm{CNVs}$, we learned that patients with $22 \mathrm{q} 11.2$ microdeletion/microduplication syndrome presented with a wide range of phenotypes concerning the abnormality of multiple systems, of which congenital heart diseases and facial dysmorphisms are the most common congenital malformations. Data on the penetrance of these recurrent CNVs is highly incomplete; some patients appear phenotypically normal, while others with the same genotype have mild to severe abnormalities. Thus, more evidence on the genotype-phenotype contributions of different 22q11.2 deleted/duplicated regions needs to be gathered.

In our summary of the abnormal 22q11.2 CNV cohort, the variants associated with proximal $(\mathrm{A}-\mathrm{D})$ region showed more severe clinical phenotypes, while those associated with the central (B-D) and distal type I (D-E/F)/type II (E-F)/type III (F-G) regions exhibited more mild or even normal features. In our laboratory, the majority of microarray cases used prenatal amniotic fluid. Due to the pathogenicity, potential terrible ending for the child, huge financial and psychological burden, most pregnant women choose odinopoeia, and only a fraction eventually give birth to their children. However, the postnatal newborns displayed various abnormalities, including neonatal dyspnea syndrome, and neonatal pneumonia. Among the 14 cases that received parental validation, 6 cases had de novo variants $(6 / 14,42.9 \%)$ and the other 8 were inherited from their parents $(8 / 14,57.1 \%$ ) (7 of the 8 from the mother). In conclusion, this study provides clinical overview of the ClinGen curation and data support for ACMG evaluation of the pathogenicity of each interval involved in $22 \mathrm{q} 11.2$ recurrent deleted and duplicated CNVs. Certainly, more evidences on the genotype-phenotype contributions of different 22q11.2 recurrent regions need to be gathered.

\section{Acknowledgments}

Funding: This work was supported by the Social
Development Public Welfare Foundation of Ningbo (Grant No. 202002N3150, 2019C50070), the First Municipal Medical and Health Brand Foundation of Ningbo (Grant No. PPXK2018-06), the Medical and Health Project of Zhejiang Province (Grant No. 2022503086).

\section{Footnote}

Reporting Checklist: The authors have completed the STROBE reporting checklist. Available at https://dx.doi. org/10.21037/tp-21-560

Data Sharing Statement: Available at https://dx.doi. org/10.21037/tp-21-560

Conflicts of Interest: All authors have completed the ICMJE uniform disclosure form (available at https://dx.doi. org/10.21037/tp-21-560). The authors have no conflicts of interest to declare.

Ethical Statement: The authors are accountable for all aspects of the work, including ensuring that questions related to the accuracy or integrity of any part of the work have been appropriately investigated and resolved. Written informed consent was provided by each pregnant woman, proband, or their parents. The study was approved by ethics board of Ningbo Women and Children's Hospital (No. EC2020-014). This study conformed to the provisions of the Declaration of Helsinki (as revised in 2013).

Open Access Statement: This is an Open Access article distributed in accordance with the Creative Commons Attribution-NonCommercial-NoDerivs 4.0 International License (CC BY-NC-ND 4.0), which permits the noncommercial replication and distribution of the article with the strict proviso that no changes or edits are made and the original work is properly cited (including links to both the formal publication through the relevant DOI and the license). See: https://creativecommons.org/licenses/by-nc-nd/4.0/.

\section{References}

1. Burnside RD. 22q11.21 deletion syndromes: a review of proximal, central, and distal deletions and their associated features. Cytogenet Genome Res 2015;146:89-99.

2. Portnoï MF. Microduplication 22q11.2: a new chromosomal syndrome. Eur J Med Genet 2009;52:88-93.

3. McDonald-McGinn DM, Sullivan KE, Marino B, et 
al. 22q11.2 deletion syndrome. Nat Rev Dis Primers 2015;1:15071.

4. Firth HV. 22q11.2 Duplication - Retired chapter, for historical reference only. 2009. In: Adam MP, Ardinger $\mathrm{HH}$, Pagon RA, et al. editors. GeneReviews®. Seattle: University of Washington, Seattle, 1993-2021.

5. Rump P, de Leeuw N, van Essen AJ, et al. Central 22q11.2 deletions. Am J Med Genet A 2014;164A:2707-23.

6. Pinchefsky E, Laneuville L, Srour M. Distal 22q11.2 microduplication: case report and review of the literature. Child Neurol Open 2017;4:2329048X17737651.

7. Cao P, Zhu X, Gu L, et al. Analysis of related phenotype of prenatal cases with copy number variations in various region of 22q11.2. Zhonghua Yi Xue Yi Chuan Xue Za Zhi. 2021;38:1055-9.

8. Rozas MF, Benavides F, León L, et al. Association between phenotype and deletion size in 22 q11.2 microdeletion syndrome: systematic review and meta-analysis. Orphanet J Rare Dis 2019;14:195.

9. Yagi H, Furutani Y, Hamada H, et al. Role of TBX1 in human del22q11.2 syndrome. Lancet 2003;362:1366-73.

10. Paylor R, Glaser B, Mupo A, et al. Tbx1 haploinsufficiency is linked to behavioral disorders in mice and humans: implications for 22q11 deletion syndrome. Proc Natl Acad Sci U S A 2006;103:7729-34.

11. Kvarnung M, Lindstrand A, Malmgren H, et al. Inherited mosaicism for the supernumerary marker chromosome in cat eye syndrome: inter- and intra-individual variation and correlation to the phenotype. Am J Med Genet A 2012;158A:1111-7.

12. Rosias PR, Sijstermans JM, Theunissen PM, et al. Phenotypic variability of the cat eye syndrome. Case report

Cite this article as: Xue J, Shen R, Xie M, Liu Y, Zhang Y, Gong L, Li H. 22q11.2 recurrent copy number variation-related syndrome: a retrospective analysis of our own microarray cohort and a systematic clinical overview of ClinGen curation. Transl Pediatr 2021;10(12):3273-3281. doi: 10.21037/tp-21-560 and review of the literature. Genet Couns 2001;12:273-82.

13. Verhagen JM, Diderich KE, Oudesluijs G, et al. Phenotypic variability of atypical 22q11.2 deletions not including TBX1. Am J Med Genet A 2012;158A:2412-20.

14. Mikhail FM, Burnside RD, Rush B, et al. The recurrent distal 22q11.2 microdeletions are often de novo and do not represent a single clinical entity: a proposed categorization system. Genet Med 2014;16:92-100.

15. Tan TY, Collins A, James PA, et al. Phenotypic variability of distal 22q11.2 copy number abnormalities. Am J Med Genet A 2011;155A:1623-33.

16. Wincent J, Bruno DL, van Bon BW, et al. Sixteen new cases contributing to the characterization of patients with distal 22q11.2 microduplications. Mol Syndromol 2010;1:246-54.

17. Ou Z, Berg JS, Yonath H, et al. Microduplications of $22 q 11.2$ are frequently inherited and are associated with variable phenotypes. Genet Med 2008;10:267-77.

18. Spineli-Silva S, Bispo LM, Gil-da-Silva-Lopes VL, et al. Distal deletion at $22 \mathrm{q} 11.2$ as differential diagnosis in craniofacial microsomia: case report and literature review. Eur J Med Genet 2018;61:262-8.

19. Fernández L, Nevado J, Santos F, et al. A deletion and a duplication in distal 22q11.2 deletion syndrome region. Clinical implications and review. BMC Med Genet 2009; $10: 48$.

20. Vyas S, Constantino JN, Baldridge D. 22q11.2 duplication: a review of neuropsychiatric correlates and a newly observed case of prototypic sociopathy. Cold Spring Harb Mol Case Stud 2019;5:a004291.

(English Language Editor: L. Huleatt) 